\title{
«La città sepolta sotto la neve». Narrazione e lirica nel carteggio Bassani-Varese
}

"La città sepolta sotto la neve ». Narration et poésie dans la correspondance Bassani-Varese

"La città sepolta sotto la neve". Narration and Lyric in the Correspondence Bassani-Varese

Lucia Bachelet

\section{OpenEdition} Journals

Edizione digitale

URL: http://journals.openedition.org/cei/3846

DOI: 10.4000/cei.3846

ISSN: 2260-779X

Editore

UGA Éditions/Université Grenoble Alpes

\section{Edizione cartacea}

ISBN: 978-2-37747-040-2

ISSN: $1770-9571$

Notizia bibliografica digitale

Lucia Bachelet, ««La città sepolta sotto la neve». Narrazione e lirica nel carteggio Bassani-Varese», Cahiers d'études italiennes [Online], 26 | 2018, online dal 28 février 2018, consultato il 27 mars 2021. URL: http://journals.openedition.org/cei/3846 ; DOI: https://doi.org/10.4000/cei.3846

Questo documento è stato generato automaticamente il 27 mars 2021

(c) ELLUG 


\title{
«La città sepolta sotto la neve». Narrazione e lirica nel carteggio Bassani-Varese
}

\author{
"La città sepolta sotto la neve ». Narration et poésie dans la correspondance \\ Bassani-Varese \\ "La città sepolta sotto la neve". Narration and Lyric in the Correspondence \\ Bassani-Varese
}

Lucia Bachelet

\section{Un dialogo «più che ventennale» ${ }^{1}$}

Quale sia stato il ruolo di Claudio Varese nella formazione di Giorgio Bassani è facile intuirlo dalla dichiarazione che l'autore ferrarese fa nel 1980 definendo il critico sassarese suo «padre spirituale, che non posso non ringraziare [...] di essere esistito, e di esistere» ${ }^{2}$. Questa è la chiosa a una lunga e duratura amicizia nata nel 1936, anno in cui il sassarese arriva a Ferrara, insieme ai cagliaritani Giuseppe Dessì e Mario Pinna, lui e gli altri professori di prima nomina provenienti dalla Normale di Pisa. La prima impressione su Bassani si può leggere in una lettera di Varese inviata a Dessì il 26 settembre di quell'anno:

C'è qui [a Ferrara] un giovane ricco-borghese ferrarese ebreo studente di lettere scrittore di novelle e abbastanza intelligente, che si è acceso del tuo Ritorno a San Silvano: e dopo averlo letto è piombato di nuovo a casa a richiedermelo ${ }^{3}$.

Nonostante l'apparente freddezza o quantomeno il distacco iniziale, fra i professori sardi e Bassani si crea ben presto un sodalizio sia letterario, nel nome di Benedetto Croce, sia politico: saranno infatti i normalisti, come è noto, a instradarlo verso posizioni antifasciste ${ }^{4}$. Nel 1938 la compagnia (che comprende anche il fratello di Giuseppe, Franco Dessì) si può dire formata e il legame fra i cinque è sancito da un racconto bassaniano pubblicato in quell'anno e significativamente intitolato Omaggio. 
Nel ritratto di se stesso e degli amici tratteggiato dall'autore, Claudio Varese è dipinto come un vecchio saggio:

Quegli che tra i cinque pareva il più anziano, anzi addirittura l'«antico» [...]; costui veniva effettivamente secondo, per età. Di statura superiore alla media, il più alto, esercitando sopra tutto quanto lo circondava come anche su se stesso una sottile e sorridente ironia, pareva che si sforzasse di dominare il meglio che poteva il violento imperio delle passioni in braccio alle quali i suoi quattro amici solevano abbandonarsi ${ }^{5}$.

3 La stima di Bassani per l'amico, più grande di qualche anno, è una costante nel rapporto fra $\mathrm{i}$ due, come dimostra l'ampio carteggio recentemente ricostituito ${ }^{6}$. La corrispondenza Bassani-Varese, o almeno quella che è arrivata fino a noi, consta di 191 pezzi e comprende l'arco cronologico che va dal gennaio del 1946, quando Bassani si è trasferito a Roma e la guerra si è ormai conclusa, al dicembre del 1973. Al centro di questo arco temporale si collocano le numerose recensioni di Varese (1954-1962), accolte nella rubrica Scrittori d'oggi della «Nuova Antologia», a buona parte delle opere bassaniane $^{7}$. Prima di tentare una doppia lettura dell'abbondante materiale a disposizione, combinando le opinioni critiche presenti nella corrispondenza con quelle contenute nelle recensioni, forniremo qualche dato utile sul carteggio che, per la sua vastità, non si potrà percorrere integralmente.

4 Lo scambio avvenuto fra i due intellettuali, ricostruito dall'Archivio degli eredi Bassani e da quello dei Varese, è costituito da un corpus, come si è detto, di 191 pezzi, fra lettere, cartoline e telegrammi, suddiviso in 145 pezzi di mano di Varese e 46 di Bassani. Essi sono distribuiti nel tempo in modo asimmetrico $0^{8}$, riassumibile in un'ideale distinzione in tre periodi: gli anni '40 di cui si hanno a disposizione pressoché soltanto lettere di Varese (69 vs 2), gli anni ' $60-70$ con quasi unicamente lettere di Bassani (3 vs 20) e una zona centrale costituita dagli anni '50 in cui è possibile ricomporre un'effettiva corrispondenza, un effettivo dialogo (62 vs 22). Benché il notevole sfasamento temporale lasci supporre che molti pezzi siano andati perduti, la disparità numerica fra i due fondi non deve stupire. La si ritrova, infatti, anche in altri carteggi, dal cui confronto si è potuto dedurre che da un lato Bassani era più accurato dei suoi corrispondenti nella conservazione delle carte, e che, dall'altro, semplicemente, scriveva meno e meno spesso ${ }^{9}$. A più missive di Varese, infatti, Bassani risponde di solito con una lunga e ragionata lettera, in cui si scusa del suo silenzio e affronta le questioni aperte dall'amico ${ }^{10}$.

5 Nello scambio epistolare fra il poeta ferrarese e il critico sardo si intrecciano vari argomenti e toni, più fili che fanno capo a tre tematiche principali ${ }^{11}$ : quella privata, il racconto delle vicende biografiche, contraddistinto da un'intonazione intima e amichevole; quella letteraria, che racchiude le vicende degli scrittori contemporanei, le loro pubblicazioni e i premi ricevuti, riguardo ai quali i due intellettuali si scambiano impressioni e opinioni; e l'ultima, più interessante da approfondire, il cui oggetto è la formazione e l'interpretazione dell'opera bassaniana. Se da un lato si può seguire la voce di Bassani che racconta il suo lavoro di scrittore, la creazione e l'evoluzione delle sue fatiche letterarie, dall'altro si può ascoltare quella di Varese che legge, da amico e critico, le opere del ferrarese, spesso allo stadio di bozze, prima ancora che vengano pubblicate, e risponde con giudizi e consigli.

6 Ma in realtà queste due voci sono indissociabili perché dialogano fra di loro in continuazione e quindi, in questo articolo, le studieremo in parallelo, in una sorta di doppia lettura, cercando di approfondire le modalità secondo cui interagiscono. Il 
periodo di interesse sarà quello degli anni ' 50 (unico decennio, come abbiamo visto, in cui è documentata una reale corrispondenza fra i due), che coincide, non a caso, con uno dei segmenti più interessanti della biografia bassaniana, quello dell'esperienza di redattore e della composizione delle prime opere. Tale scelta permetterà di entrare nell'officina poetica' di Bassani e venire a conoscenza di modelli, progetti e ripensamenti finora poco o per nulla noti; e consentirà, parallelamente, una lettura comparata fra il carteggio e le recensioni che proprio in quel decennio Varese inizia a pubblicare, e che ora, alla luce dello scambio epistolare, potranno dire qualcosa di nuovo sulla ricezione varesiana di Giorgio Bassani.

\section{Nascita di uno scrittore joyciano. La genesi de «Gli ultimi anni di Clelia Trotti»}

7 Sebbene quasi tutti i racconti e i romanzi bassaniani degli anni ' 50 siano in vario modo citati e analizzati nel carteggio, il caso de Gli ultimi anni di Clelia Trotti dimostra efficacemente il ruolo paradigmatico della corrispondenza Bassani-Varese, la doppia interpretazione di cui si accennava nel paragrafo precedente: solamente per la quarta storia ferrarese, infatti, è possibile assistere all'intero iter compositivo, interpretativo e recensorio, facendo parlare le carte - quasi - da sole. Percorreremo, dunque, il carteggio e le recensioni lungo gli anni 1953-1955, periodo in cui si possono mettere a fuoco con chiarezza le tre fasi del racconto (composizione; interpretazione; critica), attingendo, di tanto in tanto, a episodi precedenti o successivi.

\section{Composizione}

8 Conclusa Una lapide in Via Mazzini, letta e approvata da Varese, poi pubblicata su «Botteghe oscure» nell'ottobre del 1952 e prossima ad essere accolta nella silloge La passeggiata prima di cena (aprile 1953) ${ }^{12}$, Bassani dà il via a un «racconto nuovo», annunciato in una missiva a Varese del 3 gennaio 1953:

Sto lavorando - ma sono ancora all'inizio - a un racconto nuovo. Se riuscissi a impostare il primo capitolo entro l'inverno, potrei pensare di finirlo quest'estate. Ma gli inizi, come al solito, sono spaventosamente difficili ${ }^{13}$.

Come indica una successiva lettera del 29 luglio, Bassani non riuscirà, però, a rispettare il termine dell'estate:

Sto scrivendo con grande fatica, in mezzo al lavoro cinematografico e al resto, un quarto racconto: quello dell'Alda Costa. Spero, in montagna, di portarlo avanti. Se riuscissi a finirlo prima dell'inverno, potrei dire di non aver buttato via il ' $53^{14}$.

Questa volta Bassani mantiene i suoi propositi e non «butt[erà] via» il 1953. Nell'autunno di quell'anno, infatti, la prima parte del racconto è pronta: durante un viaggio a Ferrara, l'autore ne propone la lettura all'amico ("Quando venni in ottobre a leggerti il primo capitolo e una piccola parte del secondo») $)^{15}$. Il resto deve essere stato composto più tardi, fra i mesi di novembre e dicembre, se nel gennaio del 1954 Bassani scrive a Varese:

In quest'ultimo mese sono stato talmente preso da I funerali di Clelia Trotti, finiti ieri, se Dio vuole, che sono vissuto - come diceva Pasquali - come dentro un tubo, senza la possibilità, letteralmente, di occuparmi di niente altro. Il racconto è venuto alquanto più lungo dei precedenti (una cinquantina di pagine) e ho l'impressione che sia buono. Ora lo copierò. Poi spero di rinnovare il rito, a casa tua, della lettura 
davanti a te, Carmen, Nino e Giovannelli, come per Geo Josz, ricordi? Se non potrò muovermi, te lo manderò ${ }^{16}$.

11 Il ferrarese non riuscirà, però, a fare ritorno nella città natale e a «rinnovare il rito»; si dovrà accontentare di spedire il dattiloscritto e di aspettare una risposta - per nostra fortuna cartacea.

\section{Febbraio 1954. Interpretazione}

La risposta di Varese non tarda ad arrivare: l'11 febbraio 1954 il critico invia una lunga lettera vergata fronte-retro - che riporteremo, per brani, quasi integralmente - e in cui propone una chiave di lettura della nuova storia ferrarese:

Caro Giorgio,

Ho letto subito la tua Clelia Trotti; ed è stata una lettura doppia: insomma una linea ferrarese-cronachistica, un gusto di rivedere sulla scena, sia pure tracciate e deformate dall'arte, le persone che nell'apparenza quotidiana della vita apparivano o appaiono tra Corso Giovecca e Fondo Banchetto non può non accompagnarci nella lettura.

Ma una seconda lettura mi ha reso più interessante la pagina - oltre la cronaca -: il nucleo del racconto, il passaggio da Bottecchiari a Rovigatti, alla signora Codecà sino alla Trotti, il giungere a poco a poco alla conoscenza della Clelia mi pare tra le cose migliori di quell'arte intuizione della realtà, di cui parlavo nel mio articolo ${ }^{17}$.

Dunque, da un lato, il realismo della rappresentazione di Ferrara (ancora $\mathrm{F}^{*}$ ) e dei suoi variegati abitanti che percorrono le vie della città, fra il corso principale e via Fondo Banchetto, la via della maestra Clelia Trotti. Dall'altro, l'arte del lento avvicinamento, per fasi, al nucleo del racconto, alla protagonista della storia: solo nel terzo capitolo il lettore ne fa la conoscenza diretta, solo dopo aver assistito al funerale, aver ascoltato $\mathrm{i}$ vaghi accenni di Bottecchiari, i racconti del ciabattino Rovigatti e i dinieghi della signora Codecà. Varese definisce questa arte una «intuizione della realtà» di cui egli ha già parlato «nel suo articolo», ossia nella recensione alla raccolta La passeggiata prima di cena pubblicata in quello stesso febbraio del 1954. In quella occasione, la prima recensione a Bassani, il critico aveva messo a punto una linea interpretativa della narrativa bassaniana che sarebbe rimasta una cifra stilistica della critica futura, «il criterio» come scrive lui stesso «per guardare a quanto Bassani sinora ha scritto e a quanto ancora scriverà» ${ }^{18}$. Ne citiamo un estratto:

In Bassani questa ricerca di indagine morale, questo interesse vivo per la realtà, questa rinuncia all'evasione e al lirismo solitario che abbiamo visto caratteristica di molti scrittori contemporanei, e in particolare di questo gruppo di scrittori, si risolve in tono poetico. Il racconto matura in lui con un'esigenza intima e la conoscenza del mondo che esso ci prospetta diventa a un certo punto necessariamente conoscenza lirica e intuitiva, con uno scioglimento e una soluzione più completa di quello che non avvenisse in Rigoni, nella Ortese e nel La Cava e nello stesso primo Pavese. Bassani ha un interesse diretto e vivo per le cose del suo tempo, ma il suo interesse, le sue preoccupazioni civili, i ricordi politici, maturano nella cultura e nel giudizio storico e, in questa maturazione, si trasformano in fantasia ${ }^{19}$.

Nella prospettiva varesiana, Bassani ha come obiettivo principe l'analisi, l'interpretazione della realtà. A questo fine, però, lo scrittore utilizza strumenti originali rispetto ai suoi contemporanei: egli rinuncia sia al «lirismo solitario» di molti scrittori del suo tempo (i riferimenti sono a Notte di festa di Pavese, I caratteri di La Cava, Il sergente nella neve di Rigoni Stern e Il mare non bagna Napoli di Ortese, le cui recensioni, 
sempre a cura di Varese, precedono quella alla Passeggiata), sia alla pura cronaca: «Non direi perciò [...] che il suo ultimo racconto, Una lapide in Via Mazzini, sia ancora e troppo mescolato di realtà $)^{20}$, scrive più avanti il critico ${ }^{21}$. La novità di Bassani consiste, dunque, nel risolvere «in tono poetico» la realtà degli anni del fascismo, nell'adoperare una «conoscenza lirica e intuitiva» che gli permette di raccontare i fatti avvenuti tramutandoli in un'opera d'arte. Varese è riuscito a cogliere, già nelle prime tre storie ferraresi, la capacità dell'autore di far intuire la realtà, quell'arte che ritrova più tardi anche in Clelia Trotti e a cui si richiama nella lettera che abbiamo iniziato a leggere.

Se, nel complesso, la lettura del quarto racconto ha suscitato l'approvazione del critico e il richiamo a una poetica dell'intuizione, d'altra parte rimane in lui qualche riserva sul finale della storia. La lettera dell'11 febbraio prosegue:

Ma lo stesso autore mi ha letto una storia e mi ha parlato di una conclusione che qui non ho trovato. Mi pare insomma che soprattutto nella fine tu mi avessi detto di voler insistere su qualcosa di molto diverso, vitalistico, sensuale, contrapposto alla vita e alla morte di Clelia. Anche così il racconto comincia e finisce bene; ma quello che tu mi dicevi quel pomeriggio - mi pare di ottobre - potrebbe far pensare che tu potessi approfondire la fine. Bada, penso al programma del tuo racconto, e alla fine della quale mi parlavi: cioè alla separazione del giovane Lattes dal mondo di Clelia Trotti. È stato un peccato che tu non abbia avuto il tempo di leggere tutto il lavoro (a parte la nostra ammirazione per la tua arte da lettore-interprete); avremmo discusso subito questo punto. Se puoi, scrivimene ${ }^{22}$.

16 Varese, dopo aver ascoltato i primi due capitoli nel mese di ottobre, si aspettava evidentemente una conclusione diversa e, sapendo di poter influire sulle scelte dell'autore, si dispiace di non essere potuto intervenire prima. Bassani gli aveva parlato di una conclusione vitalistica, di una separazione di Bruno dal vecchio mondo socialista di Clelia Trotti e da un antifascismo con cui non era riuscito a fraternizzare. Varese immaginava, forse, una partecipazione nuova e originale di Bruno alla lotta politica, quella che Bassani aveva vissuto in quegli stessi anni impegnandosi con il Partito d'Azione.

Due giorni dopo, il 13 febbraio 1954, arriva la risposta di Bassani: due pagine fittamente dattiloscritte, che meritano di essere analizzate passo per passo.

Carissimo Claudio,

Grazie della lettera che attendevo con ansia. È vero, il programma era un po' diverso, ma poi, scrivendo, la storia mi si è mutata nelle mani. Il fatto è che, quando venni in ottobre a leggerti il primo capitolo e una piccola parte del secondo, tutto mi era abbastanza chiaro, ma non il carattere di Bruno Lattes. Lo vedevo ancora - trascinato dal pezzo autobiografico del Ciabattino che avevo scritto per la radio come una specie di autoritratto. Soltanto andando avanti, mi sono reso conto che per rendere più credibile l'identificazione di $\mathrm{F}^{*}$ col ghetto della fine del III capitolo - la svolta decisiva del racconto - dovevo farlo più giovane, più fragile di quanto, forse, io non sia stato in quegli anni ${ }^{23}$.

Per capire come e perché Bassani abbia voluto modificare il carattere di Bruno, e di conseguenza il finale del racconto, è necessario fare un passo indietro al «pezzo autobiografico del Ciabattino». Il 9 settembre 1951 Bassani aveva chiesto a Varese:

M'hai sentito ieri sera alla radio? Ho scritto una specie di racconto su commissione, sui ciabattini di Ferrara: e questo lavoretto mi ha ridato un po' di fiducia. Ho abbozzato il personaggio di un ciabattino che effettivamente stava nella piazza di S. Maria in Vado: ed ora, da qualche giorno, lo sento talmente vivere in me che vorrei mettermi subito a dargli uno sfondo più ampio, raccontando gli anni 1939-43, e la mia esperienza politica ${ }^{24}$. 
Il giorno precedente Bassani aveva letto alla radio, per il programma della RAI Scrittori al microfono. Arti e mestieri, un racconto - in prima persona - intitolato Lavoro da ciabattino ${ }^{25}$. Che il racconto, pubblicato due mesi più tardi sul "Giornale» con il titolo Il signor Rovigatti, sarebbe stato rielaborato e utilizzato per Clelia Trotti è un fatto già noto ${ }^{26}$; non altrettanto noto, però, è il tipo di legame fra i due racconti, il fatto che il Ciabattino contenesse in nuce l'idea di un racconto più lungo sull'esperienza politica negli anni della guerra, ossia l'idea originaria di Clelia Trotti. L'iniziale taglio autobiografico della quarta storia ferrarese sembra confermato dalla scelta del nome Bruno per il protagonista: Bruno Ruffo, infatti, è la falsa identità che Giorgio Bassani ha assunto dal 1943 alla fine della guerra per sfuggire alle persecuzioni ${ }^{27}$.

Dopo aver scritto i primi capitoli di Clelia Trotti, però, Bassani rifiuta l'identificazione con Bruno per ragioni che spiega nelle righe successive della lettera:

Se avessi fatto di Bruno un tipo più responsabile, quello che Clelia Trotti, nel suo vaneggiamento patetico, sognava che egli fosse, l'avrei dovuto fare assai più corazzato contro i rimpianti dei flirts borghesi, per es., spostando in tutt'altro senso l'impianto generale del racconto. L'inattualità patetica di Clelia Trotti sta anche in questo: nel non riconoscere in Bruno - lei, temperamento romantico, ottocentesco, flaubertiano, per intenderci - un tipico figlio del novecento, un infantilmente imbronciato piccolo decadente. D'altra parte, toccato il fondo della situazione con l'immagine della città sepolta sotto la neve, al termine del capitolo III, non ti pare che ogni sviluppo successivo, nel senso della partecipazione attiva di Bruno alla lotta clandestina, fosse già scontato, bruciato ${ }^{28}$ ?

21 Per ragioni narrative, di struttura e funzionalità della storia, Bassani rinuncia, infine, a quella «specie di autoritratto» da cui il racconto era nato: perché l'opposizione costitutiva della narrazione tra il romanticismo di Clelia e il decadentismo di Bruno funzioni, il secondo non può reagire agli eventi della Storia, ma deve rimanere un mediocre e anonimo sconfitto. Non si spiegherebbero altrimenti $\mathrm{i}$ «rimpianti dei flirts borghesi», ossia l'invidia di Bruno verso le due coppie di giovani ariani, i primi in Vespa, i secondi in bici, che aprono e chiudono il racconto nella piazza della Certosa e che entrambe le volte distraggono Bruno dalla figura di Clelia, che si tratti del suo funerale o di lei in persona. E si sarebbe dovuta modificare la fine del terzo capitolo, il momento in cui, «con l'immagine della città sepolta sotto la neve», Bruno capisce definitivamente che non c'è possibilità di evasione:

Non c'era che una via: quella che portava tutti, nessuno escluso, incontro a un futuro che a nessuno era più dato di poter scegliere. E allora, se la tagliola era ormai scattata, allora tanto valeva continuare così, partecipando volenterosamente, non fosse altro che per pietà di sé e di loro, ai sogni da carcerati - ai giochi solitari, ai disperati passatempi a cui ognuno per conto suo si abbandonava nella solitudine della sua cella - dei propri compagni di viaggio ${ }^{29}$.

Modificato il carattere di Bruno, anche il finale non poteva più essere quello che Bassani aveva prospettato in ottobre. La lettera del 13 febbraio continua:

$\mathrm{Da}$ ultimo, anche il progetto finale vitalistico, sensuale, mi è sembrato improvvisamente inutile, poco originale, e, soprattutto, troppo facile. Un'evasione di quel tipo, avrebbe fatto di Bruno un personaggio diverso, un intellettuale, un artista, proponendolo, alla fine, in primo piano, a suo modo vincitore della pauvre Clelia. Mentre è bene che, anche per lui, il prezzo della sopravvivenza sia, in fondo, la mediocrità, quasi l'anonimità, qualcosa di simile alla conservazione «in cera», all'efficienza da manichini, che è toccata a tutti gli altri presenti ai funerali della vecchia socialista ${ }^{30}$. 

noioso"») $)^{35}$ e il conseguente rifiuto per «Botteghe oscure», dove erano state pubblicate le prime tre storie ferraresi ${ }^{36}$, Gli ultimi anni di Clelia Trotti sono accolti da Longhi e dalla Banti nella rivista «Paragone» nell'aprile del $1954^{37}$. Qualche mese dopo, nel gennaio dell'anno successivo, il racconto viene pubblicato anche in volume, senza alcuna variante sostanziale, per la casa editrice Nistri-Lischi, nella collana narrativa diretta da Niccolò Gallo. Ed è a questa edizione che Varese scrive la sua recensione, accostandola a quella de I passeri dell'amico Giuseppe Dessì (pubblicati nella stessa collana di Clelia Trotti), della raccolta di saggi Di giorno in giorno di Emilio Cecchi e del romanzo Paolo il caldo di Vitaliano Brancati ${ }^{38}$.

Nell'articolo della «Nuova Antologia» (ottobre 1955) Varese approfondisce la funzione dell'elemento lirico nello svolgimento del racconto, un aspetto che, come abbiamo visto, si può definire caratteristico della critica varesiana. Ne citiamo, anche in questo caso, un estratto esemplificativo:

In questo libro, forse in modo più scoperto che altrove, più che in Una lapide in via Mazzini, racconto direi quasi perfetto ed esemplare, lo scrittore ha portato dei frammenti poetici, quasi appoggio ad una voce che, recitando, segna nella sua scansione lirica le tesi e le arsi del racconto, e tiene, nel suo cauto sapiente e commosso oscillare il filo della trama. Forse questa stessa cadenza lirica vela talvolta il segno del distacco che esiste tra lo scrittore e il personaggio principale. Gli ultimi anni di Clelia Trotti sembrano dunque affidati nel loro svolgersi al movimento di una cadenza tra lirica e moralistica, quasi alla voce di un narratage, quella voce cioè che accompagna e commenta in un'opera cinematografica e talvolta teatrale e suggerisce il ritmo e il punto di vista dal quale guardare ${ }^{39}$. 

quella dello scrittore che commenta liricamente il racconto (segnalando, fra l'altro, la distanza tra l'autore e il protagonista, un distacco che Bassani aveva ben sottolineato nelle lettere che abbiamo letto). Nelle righe seguenti il critico insisterà sulla capacità bassaniana di adoperare le forme liriche non solamente a chiosa del testo ma in una funzione propriamente narrativa: «I fatti, i sentimenti, i personaggi le loro azioni e i loro pensieri non soltanto si illuminano, ma anche trovano il modo di svolgersi e di girare nella trama: così a poco a poco si costruisce il racconto».

Nelle recensioni successive Varese tornerà su questa tipologia di 'commento narrativo', definendolo «una specie di contrapposizione e contrappunto lirico, una forma come già si è detto per sostenere con la lirica, col commento, il racconto o addirittura certe volte di sostituirlo» e opponendolo a un secondo tipo di commento, a quella «eco sociale di avvenimenti e atteggiamenti, quasi un rimando, una rispondenza esclamativa all'azione, o alla presenza del personaggio da parte dell'ambiente quotidiano, nel quale si inserisce, quasi un coro dissimulato e interrotto» ${ }^{40}$. È nel perfetto intreccio fra le due voci che risiede, per Varese, l'elemento di originalità della narrativa bassaniana, «simbolic $[a]$ e insieme realissim $[a]$ », che persisterà anche nei romanzi: Athos Fadigati «viene descritto con le parole e col giudizio dell'autore, e attraverso un leggero ammiccare, con le parole, con i giudizi e con la mentalità della gente, dell'opinione più diffusa, più autorevole e più conformista della città»" ${ }^{41}$, nel Giardino dei Finzi-Contini «il linguaggio lirico, le pagine di poesia così frequenti, brillanti, tenere, giuocano dialetticamente con la prosa più discorsiva o addirittura più piatta che ripete, ricalca, anzi quasi prolunga la realtà quotidiana e la sua eco» ${ }^{42}$.

La critica varesiana su Clelia Trotti suscita nuovamente un'auto-interpretazione di Bassani, che 'risponde' anche in questo caso con una lunga lettera dattiloscritta, datata 31 ottobre 1955. La recensione e la lettera possono essere lette in parallelo: esse costituiscono un vero e proprio dialogo che quasi non necessiterebbe annotazioni a margine. Seguendo l'ordine delle impressioni bassaniane, la prima - positivaconcerne il confronto con l'amico Dessì:

Il suo racconto si svolge in direzione opposta a quella di Dessì; il suo personaggio, a differenza dei personaggi di Dessì, pare voglia piuttosto togliere che non aggiungere alla realtà: il tempo nei Passeri chiariva e arricchiva le cose invece qui il tempo ogni cosa fiacca e stravolge; e la realtà è ingloriosa e gretta: la realtà era questa, inutile illudersi (p. 67), la realtà, cioè, disgregata e infiacchita, delle piccole miserie che circondano e soffocano, o almeno sembra, Clelia Trotti ancora viva, ma umiliata e stretta nella casa dei parenti, sotto la sorveglianza della polizia della dittatura, e Clelia Trotti morta, tra le miserie, le ambizioni, le distrazioni e l'indifferenza di una società comunque non eroica o non più eroica ${ }^{43}$.

Ho letto la tua recensione, anzi il tuo bellissimo articolo. Mi pare che in generale tu abbia detto cose molto giuste. [...] Finissima l'analisi dei Passeri, e particolarmente azzeccata la osservazione della divergenza che tu trovi tra il modo di Beppe e mio di metterci a confronto con la realtà ${ }^{44}$.

Meno «azzeccat[o]», invece, secondo Bassani, è il richiamo alla poesia crepuscolare:

Punti di appoggio del racconto sono le pause e le soste nelle quali la voce si abbandona a un'esclamazione poetica, e - tanto per fare richiami di comodo - a un'ironica e pur commossa elegia della vita, alla Guido Gozzano o al senso e al prestigio assoluto e vitale dell'esistenza, alla Saba ${ }^{45}$.

Quanto a Clelia Trotti, in particolare, perfetto il richiamo a Saba. Meno giusto, direi, quello a Gozzano. Il mondo che circonda Clelia Trotti è gozzaniano, oggettivamente, e non c'è nulla da fare: è così, né io potevo mutarlo. Ma Clelia Trotti, no, non è la 
signorina Felicita. Essa è morta, e morendo pone in iscacco Bruno Lattes, rivela a lui chi egli sia. Gozzano conserva sempre un margine di compiacimento estetistico. Bruno Lattes sa di essere, alla fine, non diverso dalla «squadra di nullità» a spese delle quali pensava, all'inizio, di poter sogghignare a suo piacimento ${ }^{46}$.

Il richiamo a Gozzano induce Bassani a ritornare sul carattere mediocre e anonimo di Bruno e a proporre, di nuovo, un'assimilazione del protagonista ai partecipanti al funerale della maestra socialista («qualcosa di simile alla conservazione "in cera" ${ }^{47}$ aveva scritto nel febbraio del 1954). La «squadra di nullità» richiama le autorità politiche di Ferrara che aprono il corteo funebre nel primo capitolo: «Ebbene che cos'erano, costoro, curvi e dimessi come nel complesso marciavano, se non, tutti insieme, una piccola squadra di nullità? $\aleph^{48}$. Per ribadire il destino fallimentare di Bruno, Bassani chiude la lettera con una sorprendente dichiarazione poetica, rivelando apertamente il suo debito con I morti di Joyce:

La storia del pomeriggio di Bruno Lattes in Piazza della Certosa non è che la storia della consapevolezza di un fallito. Ricordi «I morti» di Joyce? Io volevo fare di Bruno Lattes un personaggio simile a quello del marito-professore di quel racconto meraviglioso. C'è un altro punto del racconto di Joyce che avevo presente, scrivendo. Quando Bruno torna a casa, e trova il padre nello studio, e poi guarda fuori, alla città che si va ricoprendo di neve, intendevo porlo in una situazione non diversa da quella del professore-marito quando, dopo la confessione e il pianto della moglie, si accorge, guardando fuori nella notte, che «the snow was general on all Ireland $»^{49}$.

L'autore, scrivendo, aveva dunque in mente due punti di contatto fra Bruno Lattes e Gabriel Conroy, protagonista de Imorti, due punti per nulla irrilevanti. Da un lato, l'iniziale atteggiamento di superiorità del ferrarese che pensa di «poter sogghignare a suo piacimento» a spese degli altri presenti al funerale di Clelia, richiama quello dell'irlandese che durante la festa che apre il racconto - più simile a un funerale che a una festa- percepisce di possedere una cultura superiore a quella degli altri partecipanti («He was undecided about the lines from Robert Browning, for he feared they would be above the heads of his hearers»; «What did he care that his aunts were only two ignorant old women?» $)^{50}$. Dall'altro, c'è una forte similarità nel modo in cui, per entrambi, prende forma l'epifania della vuotezza e dell'ipocrisia della propria vita: il rientro a casa di Bruno alla fine del terzo capitolo ricorda quello di Gabriel in albergo; lì, nella conclusione del racconto, la neve che aveva accompagnato l'intera novella e che ora copre tutta l'Irlanda segna, come per Bruno, l'annullamento dello spazio e del tempo, l'ingresso del protagonista nel regno dei morti ${ }^{51}$.

Grazie al perspicuo confronto con Joyce, Bassani ribadisce la centralità della conclusione del terzo capitolo, che Varese, in realtà, aveva già assimilato: egli, infatti, aveva scelto quella citazione (il momento di presa di consapevolezza «Non c'era che una via.... ${ }^{52}$ ) come finale della recensione, suggestionato, probabilmente, dalla lettera del 1954 in cui lo scrittore aveva definito «la fine del III capitolo - la svolta decisiva del racconto" ${ }^{53}$.

35 Ad ogni modo, lo scambio occasionato dalla recensione della «Nuova Antologia» si conclude, anche in questo caso, con una dichiarazione di stima:

Scusa il tentativo di giustificazione. L'articolo, ad ogni modo, è bellissimo, non so quale altro critico italiano sappia come te cogliere i particolari e mantenere al tempo stesso le giuste prospettive. Sei in forma: me ne compiaccio e ti ringrazio di cuore $^{54}$. 


\section{Postilla}

Aggiungo una breve postilla che esorbita, temporalmente, da Gli ultimi anni di Clelia Trotti ma che costituisce, a mio avviso, l'esito ultimo della corrispondenza fra i due intellettuali, il risultato degli sforzi interpretativi esaminati finora. Riprendiamo il filo dalla risposta di Varese all'ultima lettera citata di Bassani:

Carissimo,

La tua lettera era molto interessante e mi confermava e mi conferma nell'idea che tu dovresti scrivere una ragion poetica, un commentario perpetuo, alle tue opere, che sarebbe cosa utilissima e stimolante per scrittori, autori e lettori contemporanei; nonché preziosa per gli storici futuri ${ }^{55}$.

Lo sprone a scrivere un commento alle proprie opere non è una novità nelle missive di Varese. Il critico che, come abbiamo visto, conosce bene la capacità di Bassani di autointerpretarsi, lo esorta a farlo con una considerevole insistenza: non si conta il numero delle volte in cui, solo nell'arco degli anni ' 50 , Varese vi fa riferimento. Alcuni esempi, in crescendo:

Ma tu dovresti promettermi di scrivere anche contemporaneamente quella ragione poetica, che tu solo puoi scrivere e che può illuminare non solo i tuoi versi, ma anche la poesia contemporanea in genere ${ }^{56}$.

E non dimenticare la mia vecchia idea, della tua ragione poetica, dell'autobiografia mentale e sociale di un lirico moderno ${ }^{57}$ !

Quanto al libro che desidero da te, devi farlo, e non aspettare: se avessi una casa editrice te lo farei fare, tenendoti lontano per un anno dal cinema; sarebbe un libro di ragioni critiche, non autobiografico in senso stretto; e sarebbe molto, molto utile, in questo momento ${ }^{58}$.

Varese, però, non si limita alla corrispondenza privata, a cui Bassani non sembra prestare molto ascolto: dal 1957 tenta la via dell'esortazione pubblica, con la speranza - vana - di un migliore effetto. L'incipit della recensione alle Cinque storie recita:

Giorgio Bassani è uno degli scrittori contemporanei che meglio potrebbe, come Henry James, commentare la formazione delle sue opere, indicare il valore, che in esse ha avuto la struttura, contribuire direttamente come critico e non solo come scrittore che dà una autobiografia intellettuale, un giornale del suo lavoro ${ }^{59}$.

Prosegue nel 1960, nell'introduzione all'intervista su Una notte del '43:

Talvolta lo scrittore non solo è criticamente consapevole della sua opera, ma critico in proprio, come appunto Bassani. Da lui infatti aspettiamo e desideriamo quella raccolta di saggi critici che tanto potrà giovare non solo per meglio capire l'arte e la personalità di lui, ma anche motivi, problemi, difficoltà e prospettive, radici e sviluppi della letteratura moderna ${ }^{60}$.

Alla fine, però, nel 1961, Varese si arrende:

Va bene. Mi rassegno. Ma dovrò comprare un magnetofono, ad uso bassanico, per poter mettere insieme questo libro di auto critiche dell'autore delle Storie ferraresi ${ }^{61}$ ?

Dieci anni dopo Bassani pubblica la Prefazione a me stesso, il breve testo in cui illustra i fondamenti estetici della sua narrativa, «il valore, che in esse ha avuto la struttura»e, insieme, la propria idea di letteratura ${ }^{62}$. In poche parole, quella «ragione poetica» a lungo richiesta. Non abbiamo prove concrete per attestare l'ascendente varesiano sulla prosa critico-autobiografica - Bassani non vi fa nessun esplicito riferimento - ma, ora, alla luce dello scambio fra i due, credo che lo si possa decisamente ipotizzare: la Prefazione a me stesso è l'esito, non sappiamo quanto conscio, di uno stimolo all'auto- 
interpretazione, al commento delle proprie opere che ha le sue lontane radici nel magistero di Claudio Varese.

Bassani, infatti, gli aveva già rivelato tutto:

Quanto al libro autobiografico che mi consigli, certo che lo farò: ma dopo aver scritto i Finzi Contini, e gli altri quattro e cinque romanzi e romanzetti che mi pullulano per la testa. Quando sarò vecchio, insomma, e non avrò proprio più altro da tirar fuori dal serbatoio. A paragone dei predetti romanzi e romanzetti tutto il resto, per ora, mi sembra vanitas vanitatum... ${ }^{63}$

\section{NOTE}

1. L'espressione si trova in una lettera di Bassani a Varese del 23 gennaio 1957.

2. G. Bassani, Testimonianza, in W. Moretti, La cultura ferrarese fra le due guerre mondiali. Dalla scuola Metafisica a «Ossessione», Bologna, Cappelli, 1980, p. 216.

3. G. Dessì - C. Varese, Lettere 1931-1977, a cura di M. Stedile, Bulzoni editore, 2002, p. 143.

4. Si veda, in particolare, il capitolo di A. Roveri, L'incontro di Bassani con Rinaldi, $i$ «sardi» $e$ Ragghanti, in Id., Giorgio Bassani e l'antifascismo (1936-1943), prefazione di P. Bassani, Sabbioncello San Pietro, 2 G Editrice, 2002, pp. 23-36.

5. G. Bassani, Omaggio, in Id., Opere, a cura e con un saggio di R. Cotroneo, Milano, Mondadori, 1998, pp. 1523-1526.

6. La mia gratitudine va agli eredi Paola ed Enrico Bassani, che mi hanno permesso di svolgere un tirocinio presso l'Archivio Bassani di Parigi per la catalogazione e digitalizzazione del Fondo epistolario Bassani, e a Ranieri Varese per avermi consentito di consultare il consistente gruppo di lettere, conservate presso l'archivio privato di Ferrara, finora sconosciute. Il carteggio è stato oggetto di un primo studio in occasione del Convegno Bassani 1916-2016 (Roma-Ferrara, 14-19 novembre 2016), i cui Atti sono in corso di pubblicazione, dal titolo Giorgio Bassani - Claudio Varese. Idee, progetti e ripensamenti; è in preparazione, invece, la pubblicazione integrale del carteggio.

7. Si tratta dei seguenti contributi di Varese sulla «Nuova Antologia»: recensione a La passeggiata prima di cena, vol. LXXXIX, febbraio 1954, pp. 272-274; recensione a Gli ultimi anni di Clelia Trotti, vol.XC, ottobre 1955, pp. 261-263; recensione alle Cinque storie ferraresi, vol.XCII, gennaioaprile 1957, pp. 121-123; recensione a Gli occhiali d'oro, vol. XCIV, 1959, pp. 541-549; recensione a Il giardino dei Finzi-Contini, vol. XCVII, giugno 1962, pp. 262-267 (le ultime tre recensioni citate saranno accolte in C. Varese, Occasioni e valori della letteratura contemporanea, Bologna, Cappelli, 1967, con il titolo Giorgio Bassani I, II, III, alle pp. 379-397). Ai contributi sulla «Nuova Antologia» si aggiunge l'intervista Tre domande a Bassani sul film di Vancini, «Il Punto», 8 ottobre 1960 (poi in C. Varese, Sfide del Novecento. Letteratura come scelta, Firenze, Le Lettere, 1992 con il titolo Tre domande a Bassani su «Una notte del '43», pp. 311-314). Si veda G. Arbizzoni, M. Ariani e A. Dolfi, Bibliografia degli scritti di Claudio Varese, Urbino, Università degli Studi, 1986, grazie alla quale si sono potuti facilmente identificare i testi citati.

8. La corrispondenza è evidentemente e sfortunatamente incompleta: le 145 lettere mandate da Varese si distribuiscono nel seguente modo: 69 negli anni '40, 62 negli anni '50, 3 negli anni '60 e 11 senza data; le 46 inviate da Bassani appartengono, invece, 2 sole agli anni ' 40 , 22 agli anni '50, 20 agli anni '60-70, mentre 2 rimangono senza data. 
9. La stessa disparità numerica si ritrova nello scambio epistolare di Bassani con Franco Fortini e in quello con Attilio Bertolucci, come si è potuto verificare dal confronto con gli interventi di G. Litrico, Nell'officina del redattore. Il carteggio tra Bassani e Fortini (1949-1959) e di F. Erbosi, Il carteggio Bassani-Bertolucci: notizie sui testi e prove d'autore all'interno del già citato convegno Bassani 1916-2016 (Roma-Ferrara, 14-19 novembre 2016).

10. Questo genere di lettere bassaniane sono spesso dattiloscritte, ben impaginate e senza correzioni; al contrario le missive di Varese sono quasi solo manoscritte, con una grafia non sempre chiara e ordinata. Una delle rarissime volte (12 su 145) in cui Varese rinuncia alla penna, riempie la pagina di errori e aggiunge un post scriptum a mano: «Ho scritto a macchina da me: imparerò mai?» (12 febbraio 1952, Varese a Bassani).

11. Per quanto il debito di Bassani nei confronti di Varese sia soprattutto politico, tale argomento, nel carteggio, non viene toccato, se non tangenzialmente. Passato il periodo di più intensa attività e lotta antifascista, l'interesse principale negli anni '50 sembra essere, almeno nello scambio epistolare, quello letterario.

12. Si tratta di Una lapide in Via Mazzini, «Botteghe oscure», $\mathrm{n}^{\circ} \mathrm{X}$, ottobre 1952, pp. 444-479; e di La passeggiata prima di cena, Firenze, Sansoni Editore, aprile 1953, pp.129-186. Nel novembre del 1952 Varese aveva scritto una lettera dall'incipit: "Caro Giorgio, scusami se non ti ho subito ringraziato per il regalo delle tue bozze; ho riletto il tuo racconto e debbo dirti che sopra tutto l'ultima parte mi ha ancora più conquistato» (17 novembre 1952, Varese a Bassani).

13. 2 gennaio 1953, Bassani a Varese.

14. 29 luglio 1953, Bassani a Varese. Come è noto, «Clelia Trotti, la protagonista del racconto, è un personaggio immaginario. Tuttavia assomiglia molto da vicino a una vecchia maestra socialista, perseguitata per le sue idee, da me frequentata molto spesso tra il '36 e il ' 43 . Si chiamava Alda Costa» (G. Bassani, Opere, cit., p. 1327).

15. 13 febbraio 1954, Bassani a Varese.

16. 14 gennaio 1954, Bassani a Varese. A questa altezza cronologica il titolo è ancora I funerali di Clelia Trotti. Il passaggio al titolo definitivo corrisponde, probabilmente, a una maggiore implicazione del testo con la realtà politica: il tema, nel senso di soggetto psicologico, passa dalla maestra Clelia Trotti a indicare un dato momento storico: il nuovo titolo non allude, infatti, a come Clelia Trotti ha vissuto i suoi ultimi anni di vita, bensì a come la città di Ferrara ha affrontato gli anni 1939-1946: «Possibile che la guerra, gli anni nei quali lui era stato ragazzo, ed essa bambina - gli ultimi anni di Clelia Trotti! - non avessero lasciato nessun segno sulla sua fronte, nessuna ombra di consapevolezza nei suoi occhi?» (G. Bassani, Gli ultimi anni di Clelia Trotti, Nistri-Lischi Editore, Pisa, gennaio 1955, p. 30). Si veda, a tal proposito, P. Italia, Tra poesia e prosa: un percorso dal carteggio Bassani-Fortini (1949-1970), in A. Perli (a cura di), Giorgio Bassani: la poesia del romanzo il romanzo del poeta, Ravenna, Giorgio Pozzi Editore, 2011, p. 68.

17. 11 febbraio 1954, Varese a Bassani. I corsivi riportati negli estratti delle lettere non sono mai dell'autrice, ma corrispondono alle sottolineature presenti nelle originali missive dei due intellettuali.

18. C. Varese, recensione a G. Bassani, La passeggiata prima di cena, cit., p. 273.

19. Ivi, pp. 272-273.

20. Ivi, p. 273.

21. Varese, riferendosi alla recensione della Passeggiata, afferma: «Ho scritto delle cose che credo debbano essere anche più dette; per togliere l'equivoco della letteratura della tua arte» (11 febbraio 1954, Varese a Bassani).

22. 11 febbraio 1954, Varese a Bassani.

23. 13 febbraio 1954, Bassani a Varese.

24. 19 settembre 1951, Bassani a Varese.

25. Il dattiloscritto del racconto per radio è conservato presso il Fondo Bassani: trascritto dagli studenti del Liceo Ariosto di Ferrara, coordinati dall'Associazione Culturale Arch'è e dalla 
Fondazione Giorgio Bassani, esso è stato pubblicato su «La Nuova Ferrara» (4 marzo 2009, pp. 1, 32) con il titolo Il ciabattino di via Borgovado; successivamente in G. Bassani, Lavoro da ciabattino, in S. Onofri (a cura di), Uno casali olim casamentivo. Un laboratorio nel Quadrivio rossettiano, Ferrara, Collana Quaderni dell'Ariosto, $\mathrm{n}^{\circ}$ 62, 2011.

26. G. Bassani, Il signor Rovigatti, «Il Giornale», 2 novembre 1951. Si veda P. Pieri, Un poeta è sempre in esilio, in R. Antognini e R. Diaconescu Blumenfeld (a cura di), Poscritto a Giorgio Bassani. Saggi in memoria del decimo anniversario della morte, Milano, Led, 2012, pp. 445-456, in particolare p. 449.

27. La falsa carta d'identità, conservata presso gli eredi, è stata recentemente esposta in occasione della mostra Giorgio Bassani: Officina bolognese (1934-1943) (Bologna, Biblioteca Comunale dell'Archiginnasio, 28 ottobre 2016 - 18 dicembre 2016), disponibile online <http:// www.movio.beniculturali.it/ams-unibo/giorgiobassaniofficinabolognese/>. Il nome Bruno Lattes potrebbe avere origine da una combinazione fra il falso nome assunto da Bassani, Bruno Ruffo, e il cognome originario di Fortini: Franco Fortini è infatti lo pseudonimo di Franco Lattes; Fortini era il cognome dalla madre cattolica, che il critico assunse nel 1940 anche per proteggersi dalle discriminazioni razziali (A. Allegra, Tra storia e profezia. Bassani e Fortini a confronto, in E. Pirvu (a cura di), Giorgio Bassani a 10 anni dalla morte, Atti del Convegno internazionale di studi, Craiova, 14-15 aprile 2010, Firenze, Cesati, 2010, p. 13).

28. 13 febbraio 1954, Bassani a Varese.

29. G. Bassani, Gli ultimi anni di Clelia Trotti, cit., pp. 79-80.

30. 13 febbraio 1954, Bassani a Varese.

31. Le Cinque storie ferraresi (Torino, Einaudi, 1956) sono riportate in G. Bassani, Opere, cit., pp. 1583-1761.

32. G. Bassani, Gli ultimi anni di Clelia Trotti, cit., p. 34.

33. 15 febbraio 1954, Varese a Bassani.

34. L'identificazione fra l'autore e il protagonista costituirà un elemento di discussione, fin dal primo apparire del racconto: in particolare Fortini accuserà Bassani di non aver manifestato un giudizio espressamente negativo sul personaggio e che anzi i punti di vista, di Bruno e dell'autore, fossero quasi sovrapposti (F. Fortini, Lettera. Iracconti di Bassani, «Lo Spettatore Italiano», a. VII, luglio 1955, pp. 296-297); per un'analisi approfondita della questione si veda P. Italia, Tra poesia e prosa, cit., in particolare pp. 65-73.

35. G. Bassani, Opere, cit., p. 941.

36. Si tratta di Storia d'amore, «Botteghe oscure», $\mathrm{n}^{\circ}$ I, luglio 1948, pp. 93-129; La passeggiata prima di cena, «Botteghe oscure», n VII, aprile 1951, pp. 15-52; Una lapide in Via Mazzini, cit. (e anche l'ultima storia ferrarese sarà accolta dalla Caetani: Una notte del ' 43 , «Botteghe oscure», $\mathrm{n}^{\circ} \mathrm{XV}$, marzo 1955, pp. 410-450).

37. Gli ultimi anni di Clelia Trotti, «Paragone-Letteratura», vol. V, $\mathrm{n}^{\circ}$ 52, aprile 1954, pp. 43-79.

38. G. Dessì, I passeri, Pisa, Nistri-Lischi Editore, 1955; E. Cecchi, Digiorno in giorno, Milano, Garzanti, 1954; V. Brancati, Paolo il caldo, Milano, Bompiani, 1955.

39. C. Varese, recensione a Gli ultimi anni di Clelia Trotti, cit., p. 262.

40. C. Varese, recensione alle Cinque storie ferraresi, cit., p. 122.

41. C. Varese, recensione a Gli occhiali d'oro, cit., p. 548.

42. C. Varese, recensione a Il giardino dei Finzi-Contini, cit., p. 265.

43. C. Varese, recensione a Gli ultimi anni di Clelia Trotti, cit., p. 262.

44. 31 ottobre 1955, Bassani a Varese.

45. C. Varese, recensione a Gli ultimi anni di Clelia Trotti, cit., p. 262.

46. 31 ottobre 1955, Bassani a Varese. Anche nella corrispondenza con Fortini, Bassani aveva ripudiato l'assimilazione a Gozzano; si veda P. Italia, Tra poesia e prosa, cit., p. 62.

47. 13 febbraio 1954, Bassani a Varese.

48. G. Bassani, Gli ultimi anni di Clelia Trotti, ed. cit., p. 24.

49. 31 ottobre 1955, Bassani a Varese. 
50. J. Joyce, Dubliners, Oxford-New York, Oxford University Press, 2000, pp. 141, 151.

51. «Yes, the newspapers were right: snow was general all over Ireland. It was falling upon the Bog of Allen and, further westwards, falling into the waves. It was falling too upon every part of the churchyard where Michael Furey lay buried. It lay drifted on the crosses and headstones, on the spears of the gate, on the thorns. His soul swooned as he heard the snow falling through the universe and falling, like the descent of their end, upon all the living and the dead» (J. Joyce, Dubliners, ed. cit., p. 176). Questa ascendenza joyciana era stata già acutamente rilevata da Piero Pieri in P. Pieri, Memoria e Giustizia. Le «Cinque storie ferraresi» di Giorgio Bassani, Pisa, ETS, 2008, p. 227.

52. G. Bassani, Gli ultimi anni di Clelia Trotti, cit., pp. 79-80 (riportata per intero poche pagine prima).

53. 13 febbraio 1954, Bassani a Varese. La citazione sarà riportata da Varese anche nella recensione alle Cinque storie (C. Varese, recensione alle Cinque storie ferraresi, cit., p. 122).

54. 31 ottobre 1955, Bassani a Varese. L'entusiasmo di Bassani sarà ancora maggiore in seguito alla recensione alle Cinque storie: «Ho letto il tuo articolo, e la nota precedente, e non so, davvero, come ringraziarti. Tu sei sempre molto buono, con me; mi hai seguito sempre, mi hai sempre spronato a fare. E se qualcosa ho fatto e farò lo debbo anche a te, alla tua amicizia ormai più che ventennale. Il tuo articolo mi è piaciuto in modo particolare per la straordinaria chiarezza, per la semplicità ed efficacia con la quale descrivi, e insieme penetri e spieghi, lo stile e la sostanza dei racconti» (23 gennaio 1957, Bassani a Varese).

55. 26 novembre 1955, Varese a Bassani.

56. 10 settembre 1951, Varese a Bassani.

57. 12 febbraio 1952, Varese a Bassani.

58. 25 giugno 1956, Varese a Bassani.

59. C. Varese, recensione alle Cinque storie ferraresi, cit., p. 121.

60. C. Varese, Sfide del Novecento, cit., p. 312.

61. 3 novembre 1961, Varese a Bassani.

62. G. Bassani, Prefazione a me stesso: cinque storie ferraresi, «Corriere della Sera», 4 febbraio 1971; poi con varianti e il titolo Gli anni delle Storie, in L'odore del fieno (Milano, Mondadori, 1972) e nel Romanzo di Ferrara (Milano, Mondadori, 1974) con il titolo Laggiù, in fondo al corridoio, ora in Opere, cit., pp. 935-943.

63. 21 giugno 1956, Bassani a Varese.

\section{RIASSUNTI}

L'articolo prende in esame una sezione ben documentata dell'intenso scambio avvenuto fra Bassani e il critico Claudio Varese, relativa alla genesi de Gli ultimi anni di Clelia Trotti (1953-1955). Attraverso la lettura incrociata del carteggio inedito e delle recensioni, si considera la doppia interpretazione della scrittura bassaniana, declinata soggettivamente (Bassani, in dialogo con Varese, si interpreta) e oggettivamente (Varese, dal dialogo con lui, individua le linee critiche sulla sua opera). Lo studio costituisce un contributo alla conoscenza di progetti e modelli bassaniani finora poco noti - come Gabriel Conroy dei Dubliners per il personaggio di Bruno Lattes - e della consapevolezza di Bassani intorno alla propria poetica. 
L'essai étudie l'intense échange qui s'est déroulé entre Bassani et le critique littéraire Claudio Varese, pendant la rédaction de Gli ultimi anni di Clelia Trotti (1953-1955). La lecture croisée de la correspondance inédite et des comptes rendus de Varese dans la presse permet d'examiner la double interprétation de l'écriture de Bassani, dans son versant subjectif (Bassani, en dialoguant avec Varese, interprète sa propre œuvre) et dans son versant objectif (Varese s'appuie sur son dialogue avec Bassani pour identifier les principales lignes critiques de son œuvre). L'étude constitue une contribution à la connaissance de projets et de sources peu connues jusque-là (par exemple Gabriel Conroy des Dubliners de Joyce comme modèle pour le personnage de Bruno Lattes) et prouve la conscience que Bassani avait de sa propre poétique.

The paper examines a well-documented section of the intense exchange between Bassani and the critic Claudio Varese about the genesis of the novel Gli ultimi anni di Clelia Trotti (1953-1955). Through a crossed reading of the unpublished correspondence and the book reviews, it considers the double interpretation of Bassani's writing, both subjectively (Bassani, in dialogue with Varese, interprets himself) and objectively (Varese, by the dialogue with him, defines the criticism of his work). The study represents a contribute to the knowledge of Bassani's new literary projects and models-like Gabriel Conroy (Dubliners) for the character of Bruno Lattesand shows the Bassani's awareness of his own poetic.

\section{INDICE}

Mots-clés : correspondance, Gli ultimi anni di Clelia Trotti, Joyce, élaboration créatrice, autointerprétation

Parole chiave : carteggio, Gli ultimi anni di Clelia Trotti, Joyce, iter compositivo, autointerpretazione

Keywords : correspondence, Gli ultimi anni di Clelia Trotti, Joyce, composition, selfinterpretation

\section{AUTORE}

\section{LUCIA BACHELET}

Università Roma Tor Vergata e Sorbonne Nouvelle Paris 3 